\title{
Aspectos económicos y contables del voluntariado en las ONGs
}

\author{
Carmen Esther Falcón Pérez* \\ Juana Fuentes Perdomo**
}

\begin{abstract}
Resumen
Habitualmente, existe una correlación cuasi directa entre las organizaciones no lucrativas y el voluntariado, constituyendo uno de los rasgos definitorios de este tipo de organizaciones del denominado tercer sector. Este trabajo plantea diversas consideraciones y alternativas relacionadas con la información contable por suministrar; de igual forma se presenta su conceptualización y, por último, la valoración económica del voluntariado, considerado como un factor productivo necesario en el desarrollo de las actividades que contribuyen al logro de los objetivos de carácter social que persiguen estas entidades.
\end{abstract}

Palabras clave: organizaciones sin fines de lucro, voluntariado, valoración.

\section{Introducción}

Cada día, en cualquier país del mundo, la mayoría de las personas aporta su tiempo, sus habilidades y conocimientos sin recibir ninguna retribución monetaria a cambio. Los voluntarios son unos agentes económicos que han recibido poca atención por parte de los investigadores de las disciplinas económicas; por ello, este trabajo tiene como principal objetivo contribuir al debate y la reflexión acerca del fenómeno del voluntariado, incidiendo en sus aspectos económicos y contables.

Para lograr este fin, la metodología que se emplea en nuestro trabajo se inicia, en primer lugar, contextualizando el estudio, para ello nos referimos a la caracterización del sector no lucrativo debido a que el voluntariado constituye uno de sus

\footnotetext{
* Profesora titular, Facultad de Ciencias Económicas y Empresariales, Universidad de Las Palmas de Gran Canaria, España. Correo electrónico: efalcon@defc.ulpgc.es

**Profesora asociada, Facultad de ciencias Económicas y Empresariales, Universidad de Las Palmas de Gran Canaria, España. Correo electrónico: jfuentes@defc.ulpgc.es
} 
Carmen Esther Falcón Pérez Juana Fuentes Perdomo

rasgos más significativos; posteriormente, hacemos énfasis en la importancia que tiene el voluntariado en las organizaciones sin fines lucrativos, para lo cual tomamos en consideración que él es usuario de la información financiera y, en consecuencia, nos cuestionamos cuál es su necesidad informativa.

Como respuesta a tales necesidades informativas, más adelante analizamos detalladamente el reflejo contable y económico del voluntariado, planteando la necesidad de informar en los estados financieros de tal elemento, su conceptualización contable y la problemática de la valoración del voluntariado; para efectuar lo anterior hacemos una revisión de la literatura que se ha ocupado del tema, así como de las diversas consideraciones y planteamientos que existen al respecto; finalmente, presentamos un apartado en que se resumen las principales conclusiones obtenidas en el trabajo.

\section{Caracterización de las organizaciones sin fines de lucro}

En la actualidad, prácticamente en todos los países con cierto desarrollo económico, un sector formado por diversas entidades diferentes entre sí ha adquirido una creciente importancia; sin embargo, presentan un rasgo común: carencia de ánimo de lucro dentro del cual existen organizaciones que prestan servicios de muy diferentes tipos —en exclusividad o en colaboración con el Estado- tales como culturales, asistenciales, deportivos, recreativos, políticos, religiosos, etc., son las denominadas "entidades no lucrativas" debido a que estas organizaciones se definen por exclusión, además se contraponen a aquellas que se identifican como lucrativas, lo que puede explicitarse en la siguiente clasificación (Henke, 1992: 13):

- Entidades lucrativas puras, que generan beneficios financieros para los propietarios.

- Entidades cuasilucrativas, que generan beneficios, en forma de servicios para los miembros.

- Entidades cuasi no lucrativas, que proporcionan servicios recuperando parcialmente el coste a través de cargas a los usuarios.

- Entidades no lucrativas puras, que proporcionan servicios basándose completamente en las necesidades de tales servicios. 
Aspectos económicos y contables

del voluntariado en las ONGs

Así, pues, el conjunto de organizaciones que indistintamente denominamos no lucrativas, sin fines lucrativos o sin ánimo de lucro abarca distintas categorías, pero todas tienen en común un elemento identificador: su objetivo no es proporcionar un beneficio de carácter financiero a sus propietarios, socios o administradores; de hecho, tales organizaciones no tienen propietarios, aunque es posible que los socios se beneficien no en términos financieros, pero sí en el sentido de usuarios de los servicios que prestan.

Aunque la existencia de organizaciones sin fines lucrativos no es en absoluto novedosa, sí es cierto que cada vez cuentan con mayor presencia e impacto en los ámbitos económico y social. De esta forma, en los últimos tiempos, en España se ha producido un aumento sin precedentes del número de entidades privadas sin fines de lucro (fundaciones, asociaciones, organizaciones no gubernamentales, etc.), lo que algunos estudiosos han calificado como "el boom de las ONG". Asimismo, cada vez más personas colaboran con estas organizaciones, ya sea a través del voluntariado o de donaciones y contribuciones dinerarias; también las empresas de negocios llegan a acuerdos de colaboración con entidades no lucrativas para la realización de actividades de carácter social, deportivo o cultural; por su parte, las administraciones públicas, además de conceder subvenciones, delegan la prestación de determinados servicios a entes privados no lucrativos. Indudablemente, el sector no lucrativo constituye un elemento significativo de la vida pública con especiales repercusiones en el ámbito económico y financiero sin olvidar los efectos positivos en la imagen que transmiten las empresas colaboradoras y patrocinadoras.

Diversos términos se emplean en la conceptualización del sector de las entidades no lucrativas, lo que refleja distintas ópticas de análisis, según enfatizan uno u otro aspecto propio y característico de estas organizaciones (Sajardo, 1996: 3032): sector caritativo, sector independiente, sector voluntario, sector exento de impuestos, sector de organizaciones no gubernamentales, sector de economía social $^{1}$, sector intermedio, sector no lucrativo y tercer sector.

\footnotetext{
1 Como señala Coque (2003: 23): "[...] en Europa y Norteamérica se ha ido generalizando el término Nueva Economía Social, cuyos contenidos se parecen mucho a los de la Economía Popular Solidaria, de amplia aceptación en América Latina y otras regiones desfavorecidas".
} 
Carmen Esther Falcón Pérez Juana Fuentes Perdomo

Podrían utilizarse múltiples criterios para definir un concepto de organización no lucrativa y acotar así el objeto de nuestro estudio. En este trabajo, utilizaremos la definición empleada en el Johns Hopkins Comparative Nonprofit Sector Project (1992), donde se afirma que una entidad no lucrativa es aquella que presenta las siguientes características:

- Está organizada formalmente, es decir, debe estar institucionalizada.

- Es privada, lo que implica que se configura como una entidad separada del gobierno.

- Disfruta de la capacidad de autocontrol institucional, dado que dispone de órganos de gobierno propios y no controlados por entidades externas, teniendo por tanto su propia regulación.

- No reparte beneficios entre los propietarios o administradores, puesto que se parte de la hipótesis fundamental de la ausencia de lucro, por lo que -en el caso de que la organización obtuviese beneficios- nunca se distribuirían.

- Tiene un marcado grado de participación voluntaria, que está compuesta en gran medida por voluntarios, si bien no es un requisito que todos sus miembros ostenten tal condición.

Esta caracterización puede ser muy útil para identificar a las entidades no lucrativas o tercer sector, pues engloba una multitud de organizaciones de muy diversa índole, por ejemplo, fundaciones, clubes, organizaciones no gubernamentales y asociaciones. No obstante, también ha de señalarse lo heterogéneo que resulta el conjunto de las entidades sin fines lucrativos, pues desarrollan sus actuaciones en distintos campos como cultura, deporte, educación e investigación, salud, medioambiente, cooperación internacional, servicios sociales e inserción social, constituyendo una actividad económica y social de indudable importancia.

Para cuantificar esta relevancia, algunas cifras pueden ofrecer una imagen de la magnitud del tercer sector en España; así, según Ruiz (2000, 259), en 1995 el número de entidades superaba el cuarto de millón, con más de veinte millones de cuotas de socios y más de once millones de socios registrados. Además, el gasto total del tercer sector ascendía en la fecha señalada al 4.61\% del PIB (sin incluir la contribución del voluntariado), elevándose hasta el 5.87\% del PIB si se incorporase el trabajo voluntario. 
Aspectos económicos y contables

del voluntariado en las ONGs

Otro elemento que confiere importancia al sector no lucrativo es su capacidad de generar empleo; así, según el Centro de Estudios Económicos de la Fundación Tomillo (2000: 54), el número de trabajadores sólo en el ámbito de acción social supera el millón, de los cuales unos 280,000 son asalariados; expresado en términos comparativos, 2 de cada 100 asalariados españoles trabaja en una ONG de acción social. Además, el sector emplea preferentemente a ciertos colectivos, que habitualmente tienen mayores dificultades para encontrar empleo, como mujeres, jóvenes y discapacitados. En muchas ocasiones, estas entidades se dedican a la creación de empleo para colectivos en riesgo de exclusión, constituyéndose en cooperativas o empresas de inserción social (Coque, 1999 y 2000).

Así, pues, el sector no lucrativo constituye una parte significativa de la realidad económica y social española que, sin embargo, no es suficientemente conocida. Por ello, conviene señalar cuáles son los rasgos que caracterizan a estas organizaciones, destacando aquellos que las identifican y confieren de entidad propia como son sus fines y objetivos.

La ausencia de ánimo de lucro es, sin duda, el aspecto clave de estas entidades; ello no significa que estas organizaciones no puedan obtener un beneficio en el desarrollo de sus actividades, sino que dicho beneficio no puede distribuirse entre los propietarios o administradores de la entidad. El objetivo de estas organizaciones no es alcanzar un excedente repartible entre los socios, como ocurre en cualquier empresa de negocios; al contrario, la finalidad de la entidad es lograr otro tipo de beneficio, no de carácter económico, sino de naturaleza social, humanitaria, educativa, cultural, ecológica, etcétera.

Los fines que persiguen estas organizaciones, por su especial naturaleza, son dificilmente compatibles con los objetivos clásicos empresariales de maximización del beneficio; por ello, en muchas ocasiones, estas entidades desarrollan sus actividades en la prestación de servicios que — por su falta de rentabilidad— no son cubiertos por las empresas de negocios; es el caso, por ejemplo, de determinadas manifestaciones artísticas y culturales. De igual forma, se produce con cierta frecuencia en el sector no lucrativo la realización de actuaciones propias de los servicios públicos, como los de asistencia social, generalmente en el marco de acuerdos de colaboración, concierto o similar entre la administración pública y las entidades no lucrativas. 
Carmen Esther Falcón Pérez Juana Fuentes Perdomo

Estas especiales relaciones que se establecen entre las organizaciones sin fines lucrativos y las administraciones públicas constituyen uno de los factores característicos del tercer sector. Además, estas entidades pueden disfrutar de determinados beneficios fiscales, como la exención del pago de impuestos o la aplicación de tipos tributarios inferiores a los soportados por las empresas de negocios; asimismo, reciben subvenciones para la realización de sus actividades, lo que constituye este mecanismo una de sus principales fuentes de financiación.

Por otro lado, en el sector no lucrativo es habitual que se produzcan determinados fenómenos - muy poco frecuentes en el sector empresarial- como son las donaciones, las cuales constituyen en muchas ocasiones una importante fuente de financiación de la entidad, que garantiza que la organización puede llevar a cabo sus actividades; regularmente, estas donaciones tienen carácter dinerario, pero también pueden estar constituidas por bienes, como puede ser un vehículo o una colección artística.

Un caso particular de donación, y exclusivo del sector no lucrativo, lo constituye el voluntariado por medio del cual una persona cede gratuitamente a la organización su tiempo, su talento y sus habilidades. Éste es uno de los valores que identifican al sector no lucrativo, especialmente en el área social y humanitaria, del que nos ocuparemos en el siguiente apartado.

\section{Importancia del voluntariado en las organizaciones sin fines de lucro}

Vernis señala que (2001: 39) "[...] si las asociaciones y fundaciones españolas tienen derecho a unos impuestos reducidos, a unas donaciones con ventajas fiscales para las personas o empresas donantes, y a recibir dinero de las administraciones públicas para realizar actividades o proyectos determinados, tienen que rendir cuentas no sólo a las personas y organizaciones que las apoyan, sino a la sociedad en general".

De esta manera, las organizaciones sin ánimo de lucro deben ser "responsables" de su actuación, deben responder ante la sociedad que las apoya. Esto es, han de "rendir cuentas", no sólo por una cuestión económica, sino también por una cuestión de legitimidad e identidad del propio sector no lucrativo; es una parte consustancial con su propia naturaleza de servicio a la sociedad, lo que algunos denominan el "contrato social" entre las entidades sin fines lucrativos y la sociedad en su conjunto. 
Aspectos económicos y contables

del voluntariado en las ONGs

De los diversos aspectos que deben considerarse en la rendición de cuentas, nos interesa destacar aquí el relacionado con quiénes ha de rendir cuentas la entidad no lucrativa, pues esta cuestión está directamente vinculada con los usuarios de la información. Según señala Leat (1996: 67-68), una de las diferencias entre las organizaciones lucrativas y las no lucrativas de voluntariado reside en la amplia gama de agentes ante los que éstas últimas deben ser responsables. Así, cita una extensa relación de grupos ante los que responder, como la comunidad o el público en general, los gobiernos central y local, las autoridades tributarias, los usuarios de los servicios, otras organizaciones voluntarias, diversos organismos reguladores y de supervisión, organizaciones profesionales y distintos grupos de suministradores de fondos. Estos colectivos conforman la panorámica de responsabilidades hacia el exterior, a los que habrá que añadir los de ámbito interno, como los voluntarios, el personal contratado, los directivos y gestores.

Por lo que respecta ante quiénes deben responder las entidades no lucrativas puede ayudarnos el mapa de las responsabilidades básicas de una organización sin ánimo de lucro, que adaptamos de Vernis (2001: 40) y que puede verse en la figura 1: 
Figura 1

Mapa de responsabilidades básicas de una organización no lucrativa

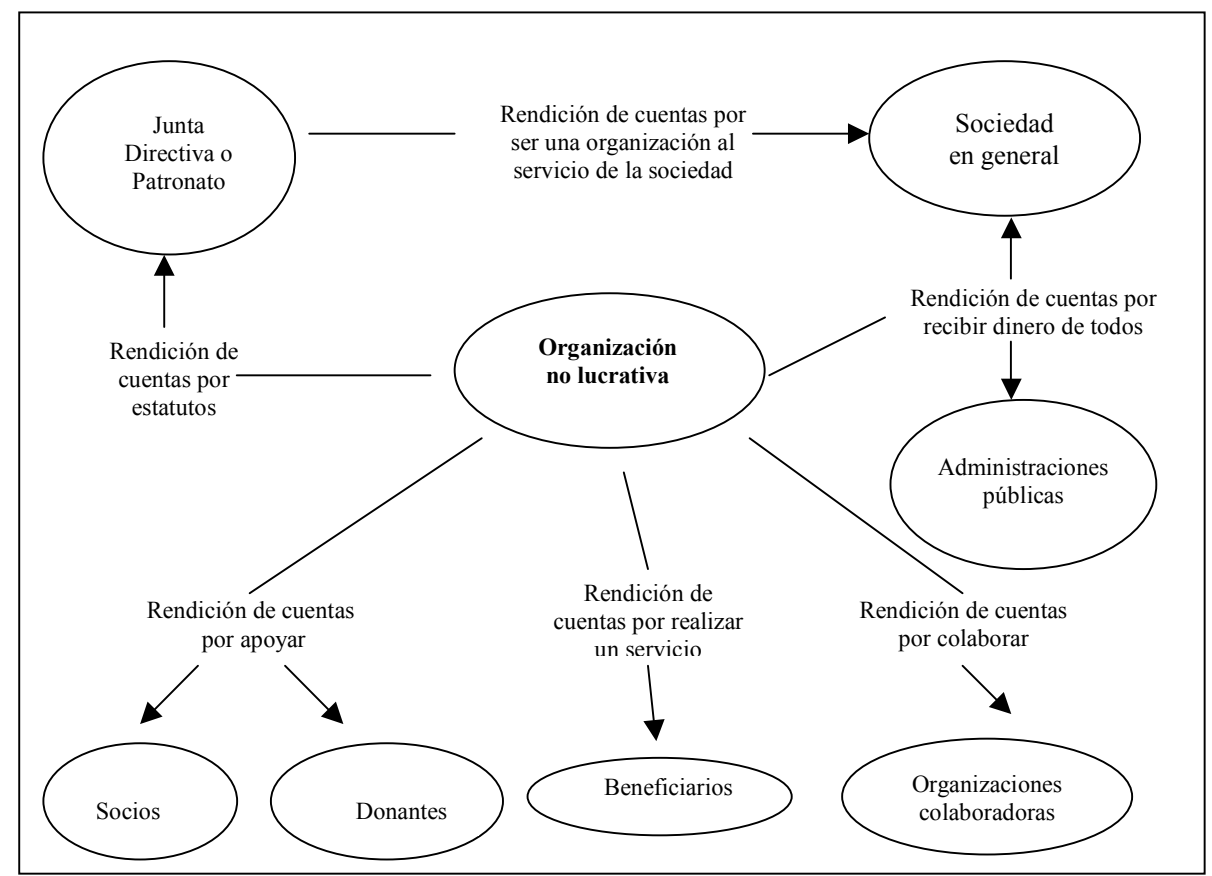

Fuente: elaboración propia, adaptado de Vernis (2001: 40)

En la figura 1 se puede observar que las entidades del sector no lucrativo deben responder de su actuación ante una amplia diversidad de agentes a quienes deben informar de diferentes situaciones, por ejemplo, desde las obligaciones de carácter legal o estatutario hasta las derivadas de la financiación pública, pasando por los compromisos adquiridos con los beneficiarios de los servicios, entre otras.

Hasta aquí nos hemos referido a la rendición de cuentas de la organización no lucrativa ante aquellos que están legitimados para exigirlo, que puede resumirse en una relación de responsabilidad de la organización frente a la sociedad. No obstante, también ha de tenerse en cuenta la especial responsabilidad que asumen (o que deben asumir) los gestores de estas entidades sin ánimo de lucro, que han de rendir cuentas de su actuación, en especial, del uso que se ha hecho de los recursos confiados y si se han utilizado según lo presupuestado o estipulado legal 
Aspectos económicos y contables

del voluntariado en las ONGs

y contractualmente. Al contrario de lo que ocurre en el ámbito empresarial, la actuación de los gestores y administradores de las organizaciones sin fines lucrativos no puede evaluarse en función de los beneficios obtenidos por éstas. Una cuenta de resultados que indique una alta cifra positiva indicaría que los gestores han efectuado mal su trabajo debido a que la entidad cuenta con recursos que no ha utilizado en su labor social, actuando de forma ineficiente. No obstante, esto no quiere decir que lo contrario sea cierto, pues cualquier organización, sea o no lucrativa, que obtenga continuadamente pérdidas está condenada a desaparecer. Es por ello que las organizaciones sin ánimo de lucro que quieran garantizar su supervivencia deben también obtener un cierto beneficio, que en este caso no será un fin en sí mismo, sino un medio para poder alcanzar los fines de la organización.

Por lo que respecta a los voluntarios y cooperantes, éstos constituyen unos agentes exclusivos de las organizaciones sin fines de lucro de los que carecen las empresas y los organismos públicos; como ya se ha señalado, el voluntariado se configura como uno de los rasgos más característicos y uno de los valores identificadores del sector que nos ocupa. Como señalan Vernis y otros (1998: 137): "[...] los voluntarios son el recurso humano por excelencia de las organizaciones no lucrativas. Sin personas voluntarias, la mayoría de estas organizaciones no sobrevivirían". De hecho, muchas de estas entidades se definen precisamente como "organizaciones voluntarias", pues su actividad es realizada por personal voluntario, que no recibe contraprestación por los servicios prestados. Así, las organizaciones sin fines de lucro deberán informar a sus voluntarios de aquellos aspectos de la actuación y gestión realizada que conecten con sus motivaciones altruistas y solidarias. Cabe señalar que éstos necesitan conocer cuál es el impacto que está teniendo su labor, en cierto modo, el reconocimiento de que las tareas que van desempeñando están contribuyendo al logro de los fines de carácter social que constituyen la razón de ser de estas entidades.

Excede de los propósitos de este trabajo realizar un análisis detallado de la problemática de la gestión del voluntariado, pero estimamos que ha de hacerse al menos referencia al proceso de captación, formación, seguimiento y evaluación de los voluntarios que dedican su tiempo, talento y habilidades a la realización de tareas de todo tipo, ya sea para la propia organización o para prestar servicios directos a los beneficiarios. 
Carmen Esther Falcón Pérez Juana Fuentes Perdomo

Las organizaciones que emplean personal voluntario, dado que no retribuyen monetariamente los servicios prestados, soportan menores costes de personal porque éstos se reducirían a los correspondientes al personal contratado (de hecho, este menor coste soportado por las organizaciones no lucrativas es una de las razones por las que en muchos estudios se califica a estas entidades como más eficientes que las empresas mercantiles, mientras que otros entienden que no es una cuestión de eficiencia, sino que al soportar menores costes, estas organizaciones practican "una competencia desleal"). Sin embargo, en términos económicos, estas entidades están consumiendo o utilizando recursos, por lo que cabría considerar alguna alternativa que facilitase evaluar cuál es la contribución que hacen los voluntarios a la actividad de la organización y cómo informar de tales cuestiones en los estados financieros, lo que será analizado a continuación.

\section{El voluntariado: su reflejo contable y económico}

El fenómeno del voluntariado es objeto de investigación habitualmente en disciplinas de carácter humanista, como la psicología o la sociología (véase, por ejemplo, los números monográficos de la Revista del Ministerio de Trabajo y Asuntos Sociales o Documentación Social, 2001); sin embargo, por parte de los investigadores del área económica y contable ha recibido menor atención

El voluntariado se caracteriza por su no retribución, como un servicio del que se disfruta, pero por el que no se paga y, por tanto, carece de cuantificación monetaria; por ello, habitualmente no es objeto de representación contable y no se informa de dicha realidad en los estados financieros de las organizaciones no lucrativas.

Con el objeto de clarificar la representación contable y económica del voluntariado, hemos estructurado este apartado para determinar, en primer lugar, si es preciso o no informar en los estados financieros sobre las aportaciones no dinerarias que habitualmente realizan los voluntarios; analizada y debatida esta cuestión, estudiaremos si el voluntariado puede ser conceptualizado como un activo intangible, pues entendemos que presenta ciertas características similares; $y$, por último, analizaremos distintas formas de valoración económica que se han propuesto por los estudiosos del tema, de esta manera, determinaremos, desde nuestro punto de vista, cuál sería la más idónea. 
Aspectos económicos y contables

del voluntariado en las ONGs

\subsection{La información contable del voluntariado ¿es preciso informar?}

Si los estados financieros pretenden proporcionar una información fidedigna de la situación económico-financiera y la capacidad de desarrollar sus actividades de una organización, cabe preguntarse si el voluntariado es un elemento que forma parte de la realidad económica y financiera de las entidades sin fines lucrativos.

En este sentido, desde el punto de vista económico, puede considerarse como un recurso del que se disfruta; y desde el punto de vista financiero, como un ahorro en el pago a trabajadores remunerados. Uno y otro enfoque pone en cuestión que los estados financieros facilitasen información acerca de una realidad que, sin embargo, habitualmente no es proporcionada por las entidades del sector.

La información contable relativa al voluntariado ha sido objeto de análisis por diversos estudiosos de la contabilidad de las organizaciones sin fines lucrativos. Anthony (1978) se refiere a esta cuestión como uno de los temas específicos que requerirían una particular atención desde el enfoque conceptual de la contabilidad de estas organizaciones, puesto que no existe un fenómeno equivalente en las empresas de negocios. Por su parte, Sánchez (1990: 203) señala que existe una tendencia generalizada a la no contabilización debido al problema que supone la valoración del trabajo voluntario.

Frente a la postura de no contabilizar el voluntariado, Morales (2002: 301) propone que

[...] su contabilización debería suponer el registro de un gasto dentro de la partida de gastos de personal al tiempo que se reflejaría por igual importe un ingreso operativo por subvención, si se cumpliesen determinados requisitos, tales como:

- Los servicios son significativos y sería preciso contratar personal asalariado si no fuesen prestados gratuitamente.

- Se puede cuantificar su importe.

Esta autora señala que si no se contabilizasen tales importes, afectaría negativamente a la comparabilidad de los estados financieros con otras organizaciones que remunerasen tales servicios. Además, reflejar contablemente el voluntariado proporcionaría una mejor valoración de los costes de los servicios que prestan las organizaciones no lucrativas. 
Carmen Esther Falcón Pérez Juana Fuentes Perdomo

En la normativa contable actualmente vigente en España para las entidades sin fines de lucro - que se encuentra en la adaptación sectorial del Plan General de Contabilidad de estas organizaciones — no se ha establecido ninguna regulación acerca de qué información ha de ofrecerse sobre la contribución de los voluntarios a la actividad de la organización, ni se ha establecido ningún tratamiento contable para reflejar esta problemática, a pesar de que el voluntariado es un recurso especialmente valioso que, sin duda, determina de modo fundamental la capacidad de la organización de desarrollar sus actividades propias.

Para paliar esta carencia, Socías (1999: 117-118) propone que a la memoria que están obligadas a elaborar las organizaciones sin fines de lucro (que completa, amplía y comenta la información contenida en el balance de situación y la cuenta de pérdidas y ganancias) se le debe añadir información de carácter social, en concreto la siguiente:

- Información sobre el voluntariado. Tipos de tareas que realizan, horas de dedicación y valoración a coste de oportunidad.

- Información sobre la posibilidad de valorar la producción social de la entidad, ya sea en el mercado real o en un mercado potencial a través de aproximaciones. Con base en ello, incorporar las magnitudes de resultado social, aportado y valor añadido social, magnitudes propuestas por el citado autor.

A nuestro juicio, es obvia la necesidad de informar en los estados financieros sobre un aspecto de tal importancia, puesto que el voluntariado es una parte consustancial con la propia naturaleza de muchas organizaciones sin fines lucrativos $y$, en consecuencia, la contabilidad no puede desatender tal realidad. Deben hacerse, por tanto, los esfuerzos necesarios para diseñar mecanismos que informen a los usuarios de cómo están contribuyendo los voluntarios a la actividad desarrollada por las organizaciones del tercer sector.

\subsection{Conceptualización del voluntariado como intangible}

Sin duda, el voluntariado constituye un recurso generador de valor para estas entidades, por lo que entendemos que puede analizarse si constituye un elemento de carácter intangible, que forma parte del denominado "capital intelectual", que contribuye al desarrollo de la actividad propia de la entidad, la consecución de los objetivos fijados y al cumplimiento de los fines que pretenden alcanzar. 
Aspectos económicos y contables

del voluntariado en las ONGs

Tradicionalmente, los activos intangibles no han formado parte de los estados financieros, pero en la actualidad son objeto de particular atención por parte de los estudiosos de la disciplina, al considerar que su no inclusión merma la capacidad de la información contable de ser útil para la toma de decisiones.

El capital intelectual se puede definir, siguiendo a Álvarez (2000: 437), como el conjunto de activos que, a pesar de no estar reflejados en los estados contables tradicionales, generan valor para la organización. El capital intelectual se configura por la interrelación de los siguientes tres componentes (IFAC, 1998):

- capital humano, que está compuesto por los conocimientos, habilidades, know-how, capacitación profesional, creatividad, flexibilidad, etc. de los miembros de la organización.

- capital relacional (también denominado capital clientela), que se basa en la satisfacción y fidelización de la clientela y, en general, en las relaciones con los agentes económicos y sociales que interactúan con la organización.

- capital organizacional (también denotado capital estructural), que comprende tanto el capital tecnológico (patentes, copyrights, marcas, franquicias, etc.) como el capital relativo a la infraestructura organizativa (cultura y filosofía corporativa, procesos de gestión, sistemas y redes de información, etcétera.).

En las entidades sin fines lucrativos, el capital intelectual puede conceptuarse, igual que en las empresas de negocios, como la interacción de los elementos humano, relacional y estructural, pero con las peculiaridades de unas organizaciones que no se orientan al accionista, sino a la consecución de sus fines de carácter social, cultural, asistencial, benéfico, deportivo, entre otros.

Así, por lo que respecta al capital humano, estas entidades cuentan habitualmente con personas conocedoras de la problemática de la que se ocupa la organización y fuertemente comprometidas con los fines de la misma (piénsese en las diversas asociaciones de "profesionales sin fronteras"), puesto que el asociacionismo surge precisamente por la confluencia de personas que coinciden en determinados objetivos. En multitud de ocasiones este capital humano está integrado por voluntarios y cooperantes, a los que no se les retribuye su labor, pero constituyen el elemento clave que dota de valor a la organización. 
Carmen Esther Falcón Pérez

Juana Fuentes Perdomo

Una vez identificado y delimitado el capital intelectual o activos intangibles de la organización, se plantea la cuestión de su valoración, lo que suscita diversas medidas alternativas que se encuentran en la actualidad sometidas a debate y a las que nos referiremos en el siguiente apartado.

Para finalizar la referencia que estamos realizando acerca de los activos intangibles de las entidades no lucrativas, hemos de plantearnos cómo ha de proporcionarse la información relativa a estos elementos que conforman el capital intelectual de estas organizaciones.

Por nuestra parte, creemos oportuno que dada la relevancia del capital humano, en particular el voluntariado en las organizaciones sin fines lucrativos se plantee incluirlo de alguna forma, al menos desde un punto de vista interno, puesto que es un factor imprescindible para la actividad propia de estas entidades.

\subsection{Diversas aportaciones sobre la valoración económica del voluntariado}

La cuestión de la valoración del trabajo realizado por los voluntarios suscita cierta polémica en el ámbito no luerativo, pues por un lado existen opiniones aeerca de que al expresar en términos monetarios una acción altruista lo que se consigue es precisamente el efecto contrario: minusvalorar esa actividad. En especial por parte de los voluntarios se suele considerar que la labor que realizan tiene un valor de carácter ético, solidario, altruista, de compromiso con determinados ideales y que, por tanto, expresar esta actividad en simples unidades monetarias desvirtúa el auténtico valor del voluntariado, que por definición no tiene precio.

En el lado contrario, se suele argumentar que al no asignar valor económico al trabajo desarrollado por los voluntarios, éste se hace "invisible", como puede observarse (o mejor dicho, no observarse) en las estadísticas del sector. Así, las políticas que se diseñan para el sector no lucrativo carecen de referencias fiables acerca de la labor que desempeñan los voluntarios y, en consecuencia, de la capacidad que tienen las organizaciones de llevar a cabo sus proyectos y programas. Si las entidades no lucrativas valorasen el voluntariado con el que cuentan, se dispondría de información más completa sobre los recursos de los que disponen. 
Aspectos económicos y contables

del voluntariado en las ONGs

Por lo anterior, consideramos conveniente revisar las diversas aportaciones que figuran en la literatura sobre la valoración del voluntariado y en las disposiciones legales del sector, a las que añadiremos algunas contribuciones propias.

En su trabajo pionero sobre cuestiones conceptuales en relación con la contabilidad financiera de las organizaciones sin fines lucrativos, Anthony (1978) propone la utilización del valor de mercado (fair market value) por analogía con el criterio empleado en el ámbito empresarial para valorar las donaciones de activos.

Como se había señalado anteriormente, Socías (1999: 118) plantea valorar el voluntariado al coste de oportunidad; esto es, evaluando qué coste supondría para la entidad no contar con el recurso de los voluntarios. En este caso, la entidad, si quisiera seguir prestando los servicios que constituyen su razón de ser, habría de contratar personal remunerado que sustituyese las labores que realizan sin contraprestación los voluntarios. La remuneración de este personal que habría que contratar sería el valor del voluntariado con el que cuenta la organización.

Éste es el criterio que se ha empleado en el estudio macroeconómico del sector no lucrativo en España de Ruiz (2000, 150-158), en que la cuantificación de la contribución del voluntariado se ha estimado como el importe que supondría retribuirlo a los niveles salariales vigentes en la fecha a que se refiere el estudio, 1995. Concretamente, se evalúa el voluntariado por su equivalente de empleo remunerado, lo que supondría 253,599 empleos a jornada completa, por un valor de 5,289 millones de euros. Dicha estimación se imputa como coste soportado por el sector, pues su no consideración supondría infravalorar la importancia económica del sector no lucrativo; pero, como no se remunera tal coste, dicho importe se incorpora también como ingreso por la donación en especie que realizan los voluntarios.

Como puede observarse, los planteamientos teóricos y prácticos señalados coinciden en que la valoración del voluntariado se resuelve por mecanismos similares a las donaciones.

Con un enfoque más económico, Goulbourne y Embuldeniya (2002: 2) consideran que medir el valor económico de la actividad voluntaria puede ayudar a mostrar: 
- Cuánto contribuyen los voluntarios a la organización en términos de tiempo empleado y gastos soportados.

- Cuánto invierte la organización en sus voluntarios.

- Cómo incrementan los voluntarios la capacidad de los recursos humanos de la organización.

- El rendimiento de la inversión que realiza la organización en su programa de voluntarios.

Para ello, estos autores proponen un total de ocho medidas de valoración económica del voluntariado, que giran en torno a la productividad de los recursos humanos, la eficiencia de los programas de voluntariado y el apoyo de la comunidad, lo cual figura en el cuadro 1.

\section{Cuadro 1}

\section{Medidas de valoración del voluntariado}

\begin{tabular}{|c|c|}
\hline \multicolumn{2}{|c|}{ Medidas de productividad de los recursos humanos } \\
\hline Valor estimado de la actividad voluntaria & $\mathrm{N}^{\mathrm{o}}$ total de horas voluntarias $\mathrm{x}$ tasa media horaria \\
\hline Verdadero valor añadido del personal & $\begin{array}{l}\mathrm{N}^{\circ} \text { total de horas voluntarias } \mathrm{x} \text { verdadero valor } \\
\text { horario (tasa media salarios }+ \text { beneficios sociales) }\end{array}$ \\
\hline Equivalente a jornada completa anual & $\begin{array}{l}\left(\mathrm{N}^{\mathrm{o}} \text { total de horas voluntarias / } 40 \text { horas }\right. \\
\text { semanales }) / 48 \text { semanas }\end{array}$ \\
\hline $\begin{array}{l}\text { Porcentaje de ampliación de valor del } \\
\text { personal }\end{array}$ & $\begin{array}{l}\text { [Valor actividad voluntaria / (Total de salarios } \\
\text { pagados + valor actividad voluntaria)] x } 100\end{array}$ \\
\hline \multicolumn{2}{|c|}{ Medidas de eficiencia del programa de voluntariado } \\
\hline $\begin{array}{l}\text { Ratio de inversión de la organización en } \\
\text { voluntariado }\end{array}$ & $\begin{array}{l}\text { [Valor actividad voluntaria / inversión financiera } \\
\text { total de la organización en el programa de } \\
\text { voluntariado] x } 100\end{array}$ \\
\hline $\begin{array}{l}\text { Ratio de voluntarios por personal } \\
\text { contratado }\end{array}$ & $\begin{array}{l}\mathrm{N}^{\mathrm{o}} \text { total de voluntarios / } \mathrm{N}^{\mathrm{o}} \text { total de empleados en } \\
\text { el programa de voluntariado }\end{array}$ \\
\hline \multicolumn{2}{|c|}{ Medidas de apoyo de la comunidad } \\
\hline Contribución voluntaria al capital & $\begin{array}{l}\text { Total de gastos efectuados por los voluntarios - } \\
\text { Total de los gastos que se reintegran a los } \\
\text { voluntarios + importe total donado por los } \\
\text { voluntarios }\end{array}$ \\
\hline Ratio de inversión en la comunidad & $\begin{array}{l}\text { Valor actividad voluntaria / presupuesto total del } \\
\text { programa de voluntariado }\end{array}$ \\
\hline
\end{tabular}

Fuente: Adaptado de Goulbourne y Embuldeniya (2000) 
Aspectos económicos y contables

del voluntariado en las ONGs

En relación con la normativa contable que se ha dictado en España para regular la información financiera de las organizaciones sin fines de lucro no se establece ninguna disposición sobre la valoración del voluntariado, puesto que no se exige información sobre el mismo, como ya se había indicado.

No obstante, la citada regulación contable específica establece como principio contable, de aplicación obligatoria, el del precio de adquisición en los mismos términos que para las empresas de negocios. En este sentido, la aplicación del principio del precio de adquisición puede ser problemática en lo que respecta a la valoración de las donaciones, puesto que el precio (que no el valor) de activos que se adquieren sin contraprestación alguna es un importe irrelevante o nulo. En este sentido, cabría plantearse la valoración del voluntariado como un caso particular de donación, puesto que la peculiaridad de su gratuidad difícilmente puede encajar dentro del esquema del precio de adquisición porque que no se pagan tales servicios.

Por lo que se refiere a la adquisición de activos a título gratuito, en aplicación del principio del precio de adquisición en la adaptación sectorial se establece su valoración aplicando el valor venal ${ }^{2}$, empleando como contrapartida una cuenta de ingresos a distribuir en varios ejercicios. No obstante, puesto que en el sector no lucrativo las donaciones son un fenómeno mucho más frecuente que en el mundo empresarial, la imputación de tales ingresos no se realiza a los resultados extraordinarios — como en las empresas—, sino que si la donación afecta (como es habitual) a las actividades propias de la entidad no lucrativa se incorpora como componente del resultado ordinario. Esta problemática podría aplicarse, por analogía, al voluntariado, que pudiera cuantificarse al valor venal; esto es, el precio que se presume estaría dispuesto a pagar la organización para adquirir los servicios prestados por los voluntarios.

En suma, tras la revisión de la literatura al respecto y el análisis de la regulación contable de las entidades no lucrativas, consideramos oportuno incorporar al debate ciertos planteamientos propios, que puedan contribuir a mejorar la información contable acerca del voluntariado.

2 "El valor venal de un bien es el precio que estaría dispuesto a pagar un adquirente eventual teniendo en cuenta el estado y el lugar en que se encuentre dicho bien. El valor venal se apreciará en función de la situación de la empresa y, generalmente, bajo la hipótesis de continuidad de explotación del bien" (Plan General de Contabilidad, 1990, NV.2 $2^{\mathrm{a}}$. 
Carmen Esther Falcón Pérez Juana Fuentes Perdomo

Anteriormente planteamos la consideración del voluntariado como recurso intangible de estas entidades, por lo que nos referiremos a la valoración de tales intangibles, aunque hay que indicar que en la actualidad sigue generando debate y polémica la valoración del capital intelectual. En este sentido Salas (2001: 11) opina que "[...] es deseable que los activos intangibles se reflejen en el balance según su coste de reposición, pero al mismo tiempo existen dificultades para estimar ese valor y, por tanto, el riesgo consecuente de que a través de la contabilidad se "maquillen" los resultados económicos y financieros publicados". El citado autor considera que la problemática económico-contable de los activos intangibles ha de tener una doble vertiente, por un lado proporcionar información interna que ayude a mejorar las decisiones de la gestión, lo que precisa la determinación de un coste en términos de lo que cuesta reponer los activos tangibles e intangibles necesarios para producir una corriente de beneficios; y, por otro lado, suministrar información externa que permita evaluar la riqueza creada por las empresas, principalmente por aquellos que invierten en ellas su capital, lo que requiere estimar un valor de las corrientes de beneficios presentes y futuros que se espera recibir como inversor de la empresa.

En el ámbito no lucrativo este planteamiento ha de vincular los activos intangibles, no con la obtención por parte del accionista de beneficios futuros, sino con el cumplimiento de los fines que la organización se ha propuesto alcanzar. Es por ello que consideramos que los avances que se produzcan en la cuestión de la valoración de intangibles en el ámbito de las empresas de negocios puedan contribuir también a determinar la valoración del voluntariado. Además, ha de considerarse que la argumentación que planteamos de conectar los intangibles con el cumplimiento de los fines de la organización puede ser aplicable también a las entidades públicas, como puede verse en Caba y Sierra (2000).

Las dimensiones del fenómeno del voluntariado son múltiples y complejas, pues coexisten elementos sociológicos, psicológicos, motivacionales y éticos que escapan del campo de estudio de la contabilidad financiera; pero el voluntariado también presenta una dimensión económica como recurso de amplia utilización en el sector no lucrativo; por ello, en nuestra opinión, debe cuantificarse su contribución a las actividades de las organizaciones sin ánimo de lucro.

Debemos considerar que los servicios prestados por este tipo de entidades también pueden ser ofrecidos por cualquier otra sociedad; sin embargo, y desde el punto de vista económico, a las entidades empresariales no les resulta viable por- 
Aspectos económicos y contables

del voluntariado en las ONGs

que habitualmente los servicios ofertados a una clientela suelen ser grupos o nichos de mercado con escaso poder adquisitivo; en definitiva, las organizaciones sin ánimo de lucro ayudan a la comunidad de una forma más o menos gratuita.

Ahora bien, en toda organización se debería determinar el coste del producto o servicio ofertado - $-y$ en este caso las entidades sin ánimo de lucro no son una excepción-, el cual deberá incluir todos aquellos factores productivos que han sido consumidos para que finalmente se obtenga el servicio con independencia de que sea gratuito al cliente. Basándonos en este argumento, uno de los factores productivos más importante en estas organizaciones es el factor trabajo (médicos sin fronteras, asistentes sociales, psicólogos, etc.), pues estas organizaciones tienen un contacto directo con las personas más necesitadas, lo que nos conduce a establecer diferentes alternativas en la valoración de este factor, el voluntariado, al margen de aquellos otros costes que perfectamente se pueden valorar económicamente como son los costes de captación, formación, selección y los costes correspondientes a los seguros contra riesgos de accidente y enfermedad derivados directamente del ejercicio de la actividad voluntaria.

A nuestro juicio, tras el estudio realizado, proponemos como opciones para la valoración del voluntariado:

- El coste de oportunidad, por ser el criterio más comúnmente referido en la literatura al respecto.

- La cuantificación de tales costes a través de los ingresos, como una contribución de este trabajo a la problemática de la cuantificación del voluntariado.

Si consideramos el coste del voluntariado como el coste de personal en que se debería incurrir si las entidades no lucrativas, como cualquier otra empresa comercial, contrataran la mano de obra necesaria para poder realizar las tareas propias de estas entidades, entonces estaríamos refiriéndonos al coste de oportunidad.

De esta manera, podría considerarse una especie de valor venal de los servicios prestados por los voluntarios como el equivalente monetario que tendría que retribuir la organización si tuviese que contratar dichos servicios; por ejemplo, en el caso de un voluntario que realizase labores de asistencia social para los beneficiarios de la organización, se cuantificaría su contribución como el equivalente que 
Carmen Esther Falcón Pérez Juana Fuentes Perdomo

tendría que pagar la entidad a un asistente social contratado para la realización de dichas labores. Dicho de otro modo, la entidad cuenta con un recurso que no remunera, así que podría valorarse el coste de oportunidad que se está ahorrando. Asimismo, no debemos olvidar que a pesar de que las organizaciones objeto de estudio no persiguen fines lucrativos, nos movemos en un mundo económico en el que prácticamente todo es medible económicamente $\mathrm{y}$, por tanto, todo tiene un precio.

Somos conscientes de que no siempre podrá asignarse directamente tal valor, en especial en aquellos servicios que no tienen equivalente en el mercado laboral, pues hay que recordar que en el sector no lucrativo y, en especial en el ámbito de los servicios sociales se generan "yacimientos de empleo" porque se detectan necesidades y se crean una serie de servicios nuevos que inicialmente no se facilitan por el mercado. En tal caso se podría tomar como referencia las tarifas vigentes previstas en los colegios profesionales correspondientes.

La determinación del coste sería sencilla porque habría que localizar las distintas categorías de personal requerido y el número de horas que presta sus servicios a las organizaciones no lucrativas. Anualmente, es conocido el salario mínimo interprofesional y en cuánto se diferencia según las distintas categorías profesionales y las cualificaciones correspondientes. Así, no se debe valorar económicamente de la misma forma los servicios prestados por un asistente social en su lugar de residencia que un médico "sin fronteras" que realiza su labor médica en cualquier parte del mundo.

Con ello se pone de manifiesto que -además de las tablas dispuestas legalmente para establecer el coste del personal en función de su categoría profesional, entendido como el mínimo valor-al hablar de coste de oportunidad también deberíamos cuantificar aspectos relativos a la lejanía de la residencia, peligrosidad, zona de conflictos, etc., que son elementos muy difíciles de valorar económicamente, pues se está poniendo en definitiva y en algunos casos "precio a la vida humana", pero ha de tenerse en cuenta que si no fuera por estas personas voluntarias no se prestarían este tipo de servicios.

La otra posibilidad que proponemos para cuantificar el coste del voluntariado, y que no conocemos que se haya planteado anteriormente por otro autor, vendría dada por la corriente positiva en todo resultado: los ingresos; en definitiva, valorar la producción de las entidades sin ánimo de lucro. 
Aspectos económicos y contables

del voluntariado en las ONGs

Habrá que determinar el valor de la producción en el mercado; esto es, el precio de venta de los servicios prestados por estas unidades económicas, realizado en función de un estudio de mercado, de tal forma que se pueda determinar el resultado por contraposición de los ingresos calculados directamente relacionados con las actividades para las que se constituyó la organización no lucrativa y los costes que han sido precisos para su obtención, entre los que se debería incluir está factor trabajo (y, por tanto, el voluntariado). De esta manera, si partimos de la valoración previa de los ingresos, éstos deberían coincidir con la magnitud de costes, puesto que el resultado sería nulo, máxime cuando estas entidades no persiguen ánimo de lucro, como puede apreciarse en el cuadro 2.

Cuadro 2.

Cuantificación del voluntariado a través de los ingresos

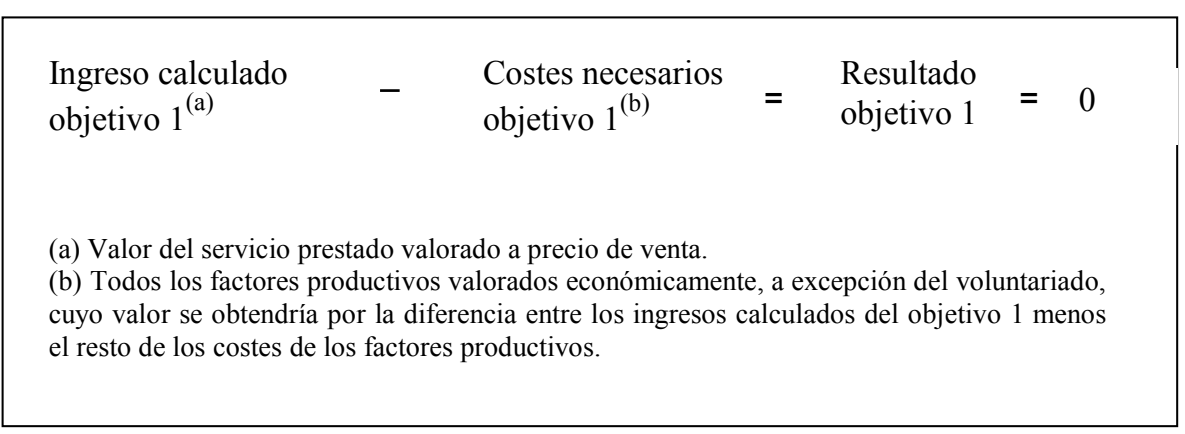

Fuente: Elaboración propia

En consecuencia, el voluntariado vendría valorado por la diferencia entre el valor de la producción (a precio de venta) y el resto de factores productivos que sí son cuantificados económicamente en el presupuesto.

A pesar de presentar esta segunda posibilidad, entendemos que se debería optar por valorar en función del coste de oportunidad en la medida que la cuantificación sería más acorde a las distintas categorías profesionales, puesto que si se valorase según los ingresos calculados se cuantificaría el coste total del voluntariado, debiéndose hacer una labor posterior para discernir cuánto correspondería a cada trabajador en función de la categoría y el número de horas prestadas. Además, han de considerarse las dificultades de valorar la corriente de ingresos porque en muchas ocasiones los servicios prestados por los voluntarios son de carácter exclusi- 
Carmen Esther Falcón Pérez Juana Fuentes Perdomo

vo que no tienen correspondencia en el mercado y, en consecuencia, la estimación de tales ingresos puede carecer de suficiente fiabilidad. En cambio, la valoración del factor trabajo es más factible acudiendo a la cuantificación a través del coste de oportunidad, asimilándolo al trabajo remunerado.

A efectos de suministrar información sobre la valoración económica del voluntariado a los socios y usuarios se podría optar por mostrarlo en un balance social, o en la memoria, de tal manera que se informara de cuánto sería el coste del servicio prestado por las entidades no lucrativas si incluyesen la valoración del voluntariado y cuánto sería el coste del servicio prestado, sin considerar la contribución de los voluntarios para efectos comparativos.

\section{Conclusiones}

Las organizaciones sin fines lucrativos no podrían existir sin las aportaciones gratuitas que realiza el factor humano: el voluntariado, cuyas repercusiones son de indudable importancia porque desempeñan su labor en todo el mundo, en muchas ocasiones prestando servicios que las empresas lucrativas no estarían dispuestas a realizar por su falta de rentabilidad. A pesar de su importancia, actualmente no existe unanimidad de criterios en torno al reflejo contable y la valoración económica del voluntariado.

Tras el estudio realizado, concluimos que el voluntariado en el marco de las organizaciones sin fines lucrativos ostenta tal importancia que los estados contables de dichas entidades deben informar sobre dicho factor productivo; de lo contrario, la información contable facilitada no podría ser comparable con la de otras entidades de carácter privado que pudieran prestar los mismos servicios. Si el voluntariado no se refleja contablemente, éste se hace "invisible" y se estaría ofreciendo una imagen incompleta de la realidad de estas organizaciones (pues aparentemente no utilizan tales recursos), que cuando verdaderamente no tendrían razón de existir si el voluntariado no prestara estos servicios. Una opción propuesta en el trabajo es conceptuarlo contablemente como activo intangible; es decir, además de que entendemos que es preciso informar, también planteamos cómo sería su consideración contable. 
Aspectos económicos y contables

del voluntariado en las ONGs

Por lo que respecta a su valoración económica, no tenemos dudas en que este recurso debe ser cuantificado, pues no hay que confundir que el servicio se preste gratuitamente con lo que significa que todo trabajo debe ser remunerado; asimismo, es importante señalar que aunque el voluntariado no tenga precio no significa que no tenga valor. Después de analizar las propuestas de distintos autores y proponer una novedosa - reconocimiento económico en función de los ingresos - creemos conveniente decantarnos por la valoración del voluntariado en función del coste de oportunidad, pues sería el criterio más idóneo porque intentaría recoger todas las características de un servicio que se presta en el mercado en condiciones similares, aunque su cuantificación en la práctica podría adolecer de una cierta subjetividad en aquellas circunstancias en que la labor de los voluntarios se desarrolla en situaciones de riesgo o condiciones extremas.

\section{Bibliografía}

ÁLVAREZ LÓPEZ, J. (2000), "Medición y valoración del capital intelectual", en Técnica Contable, No 618, junio, pp. 433-441.

CABA PÉREZ, C. y M. Sierra Fernández (2000), "Necesidad de información sobre el capital intelectual en los organismos públicos: una propuesta para su medición", en IX Encuentro de Profesores Universitarios de Contabilidad. La Contabilidad ante el nuevo milenio, Centro de Estudios Financieros, Las Palmas de Gran Canaria, Tomo 1, pp. 461-481.

CARIDE GÓMEZ, J. A. y J. F. López Paz (ed.) (2002), Ocio y voluntariado social. Búsquedas para un equilibrio integrador, Universidad de Deusto, Bilbao.

CENTRO DE ESTUDIOS ECONÓMICOS DE LA FUNDACIÓN TOMILLO (2000), Empleo y trabajo voluntario en las ONG de acción social, Ministerio de Trabajo y Asuntos Sociales, Madrid.

CHACÓN, F. y M. L. Vecina (2002), Gestión del voluntariado, Síntesis, Madrid. 
Carmen Esther Falcón Pérez Juana Fuentes Perdomo

COQUE MARTÍNEZ, J. (1999), "Los promotores de sociedades cooperativas de inserción social: análisis económico y jurídico", Revista de Estudios Cooperativos (REVESCO), Nº7, pp. 7-36.

COQUE MARTÍNEZ, J. (2000), ¿Qué son las empresas de inserción social?", en Ciclos de conferencias de interés para emprendedores y pequeños empresarios, Ayuntamiento de Gijón, Gijón.

COQUE MARTÍNEZ, J. (2003), "La nueva economía social: respuestas cooperativas", Ábaco: Revista de cultura y ciencias sociales, № 36, pp. 23-34.

DOCUMENTACIÓN SOCIAL (2001), Repensar el voluntariado, Número monográfico, $n^{\circ} 122$.

EUROPEAN VOLUNTEER CENTRE (2005), Voluntary Action in Spain, Facts and Figures, European Volunteer Centre.

GIL MENESES, J.M. (2004), "Voluntarios y remunerados, dificultades de la gestión en las ONG", Boletín CIES, junio, Nº 16.

GOUlBOURNE, M. y D. Embuldeniya (2002), Assigning Economic Value to Volunteer Activity: Eight Tools for Efficient Program Management, Canadian Centre for Philanthropy, Toronto.

HENKE, E.O. (1992), Introduction to Nonprofit Organization Accounting, $4^{\mathrm{a}}$ ed., South-Western Publishing Co., Cincinnati, Ohio.

HERRANZ BASCONES, R. (2005), Las organizaciones no gubernamentales. Un Modelo integral de gestión y control, Asociación Española de Contabilidad y Administración de Empresas, Madrid.

INTERNATIONAL FEDERATION OF ACCOUNTANTS, IFAC (1998), The Measurement and Management of Intellectual Capital: An Introduction. [disponible en internet: http://www.ifac.org] 
Aspectos económicos y contables

del voluntariado en las ONGs

LEAT, D. (1996), "Are Voluntary Organisations Accountable?", en D. Billis y M. Harris (eds.): Voluntary Agencies. Challenges of Organisation and Management, MacMillan Press, London, pp. 61-79.

MONTAGUT, T. (coord.) (2003), Voluntariado: la lógica de la ciudadanía, Ariel, Barcelona.

MORALES GUERRERO, A. (2002), La realidad económica de las fundaciones. Información contable y gestión en las entidades no lucrativas, Secretariado de publicaciones e intercambio editorial de la Universidad de Valladolid, Valladolid.

PALMER, P. y A. Randall (2002), Financial Management in the Voluntary Sector. New Challenges, Routledge, London and New York.

PUIG OLLÉ, A. (1998), "Las empresas de inserción: el papel de las fundaciones y las asociaciones en la creación de las empresas", en Revista de Estudios Cooperativos (REVESCO), Nº 65, pp. 69-72.

REAL DECRETO 1643/1990, 20 de diciembre, se aprueba el Plan General de Contabilidad.

REAL DECRETO 776/1998, 30 de abril, se aprueban las normas de adaptación del Plan general de contabilidad a las entidades sin fines lucrativos y las normas de información presupuestaria de estas entidades.

REVISTA DEL MINISTERIO DE TRABAJO Y ASUNTOS SOCIALES (2001), Voluntariado, número extraordinario.

RODRÍGUEZ CABRERO, G. (2002), "Políticas sociales y voluntariado", en $6^{\circ}$ Congreso Estatal del Voluntariado. Voluntariado y Cambio Social, Gobierno de Aragón y Ministerio de Trabajo y Asuntos Sociales, Zaragoza.

RUIZ OLABUÉNAGA, J.I. (Dir.) (2000), El sector no lucrativo en España, Fundación BBV, Bilbao. 
Carmen Esther Falcón Pérez

Juana Fuentes Perdomo

SAJARDO MORENO, A. (1996), Análisis económico del Sector No lucrativo, Tirant lo Blanch, Valencia.

SALAMON, L.M. y H. K. Anheier (1992), "In search of the non profit sector, II. The problem of classification", Voluntas, vol. 3, N 3, pp. 267-309.

SALAS FUMÁS, V. (2001), "Sobre valor y costes de los intangibles", Actualidad Financiera, $\mathrm{N}^{\mathrm{o}} 1$, pp. 3-12.

SÁNCHEZ ARROYO, G. (1990), La contabilidad en las entidades sin finalidad lucrativa, Instituto de Contabilidad y Auditoría de Cuentas, Madrid.

SEBASTIÁN HERRANZ, M. (1998), "Las personas con discapacidad y el acceso al empleo independiente", en Revista de Estudios Cooperativos (REVESCO), $\mathrm{N}^{\mathrm{o}} 65$, pp. 117-124.

SOCÍAS SALVÁ, A. (1999), La información contable externa en las entidades privadas no lucrativas, Especial referencia a las ONGs, Instituto de Contabilidad y Auditoría de Cuentas, Madrid.

VERNIS DOMÈNECH, A. (2001), "Los diferentes elementos de la rendición de cuentas en las organizaciones no lucrativas", en La transparencia de la solidaridad, Comunidad de Madrid, Fundación Lealtad, Madrid, pp. 33-50.

VERNIS DOMÈNECH, A. et al. (1998), La gestión de las organizaciones no lucrativas, Deusto, Bilbao.(A) 